\title{
Microwave Antenna Performance Metrics
}

\author{
Paul Osaretin Otasowie
}

Additional information is available at the end of the chapter

http://dx.doi.org/10.5772/48517

\section{Introduction}

An antenna is a conductor or group of conductors used for radiating electromagnetic energy into space or collecting electromagnetic energy from space. When radio frequency signal has been generated in a transmitter, some means must be used to radiate this signal through space to a receiver. The device that does this job is the antenna. The transmitter signal energy is sent into space by a transmitting antenna and the radio frequency energy is then picked up from space by a receiving antenna. The radio frequency energy that is transmitted into space is in the form of an electromagnetic field. As the electromagnetic field arrives at the receiving antenna, a voltage is induced into the antenna. The radio frequency voltage induced into the receiving antenna is then passed into the receiver. There are many different types of antennas in use today but emphasis is on antennas that operate at microwave frequencies. This chapter discusses the two major types microwave antenna which are the horn-reflector and parabolic dish antennas. In order to satisfy antenna system requirements for microwave propagation and choose a suitable antenna system, microwave design engineers must evaluate properly these antenna properties in order to achieve optimum performance.

\subsection{Definition of microwave and microwave transmission}

Microwaves refer to radio waves with wavelength ranging from as long as one meter to as short as one millimeter or equivalently with frequencies between $300 \mathrm{MHz}(0.3 \mathrm{GHz})$ and $300 \mathrm{GHz}$.

Microwave transmission refers to the technology of transmitting information by the use of radio wave whose wavelength is conveniently measured in small numbers from one meter to one millimeter.

\section{Microwave antenna}

Microwave antenna is used for radiating microwave signal into space and receiving microwave signal from space. Microwave antenna acts as a transition region between free 
space and guiding structure like a transmission line in order to communicate between two or more locations. In microwave antennas, high gains with very narrow beam width in one or more planes are required. These can be achieved with antennas of reasonable size.

\subsection{Types of microwave antenna}

There are two main types of antenna that are used in microwave links. They are:

1. A horn-reflector antenna

2. A parabolic dish antenna

\subsubsection{Horn-reflector antenna}

A horn antenna is nothing more than a flared wave-guide as shown in Figure 1. The horn exhibits gain and directivity, however its performance is improved more by using it in combination with a parabolic reflector.[1]

An open-ended wave-guide is an inefficient radiator of energy due to the impedance mismatch at the mouth, it can be improved by simply flaring the end of the waveguide. Flaring of the wave-guide ends creates a horn antenna as shown in Figure 2.

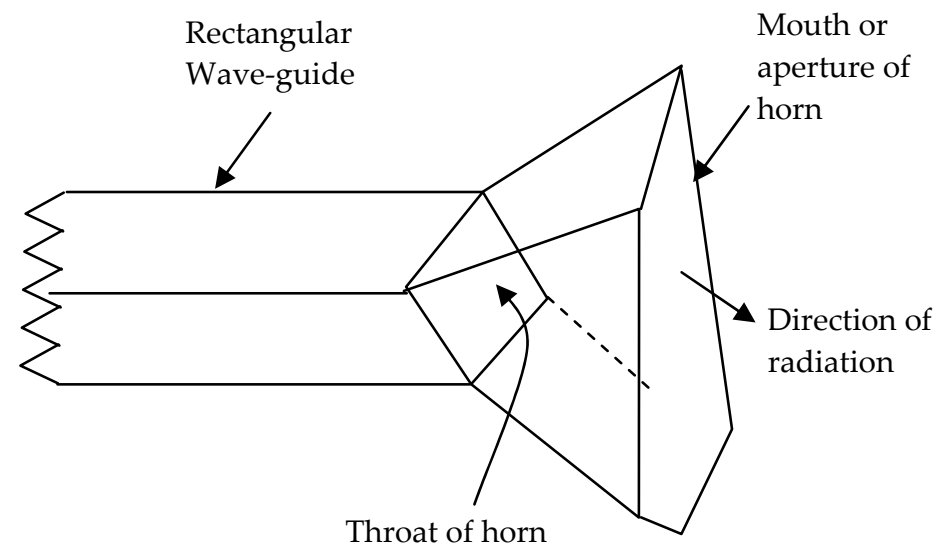

Figure 1. Basic horn antenna

Horn antennas have excellent gain and directivity. The longer the horn, the greater its gain and directivity. Different kinds of horn antennas can be created by flaring the end of the wave-guide in different ways for example flaring in one dimensions creates a sectoral horn e.g. horns flared in the $\mathrm{E}$ or $\mathrm{H}$ planes. Flaring the wave guide in both dimension produces a pyramidal horn e.g. horns flared in both $\mathrm{E}$ and $\mathrm{H}$ planes. If a circular wave-guide is used the flare produces a conical horn. These are shown in Figure 3.(a to d) 


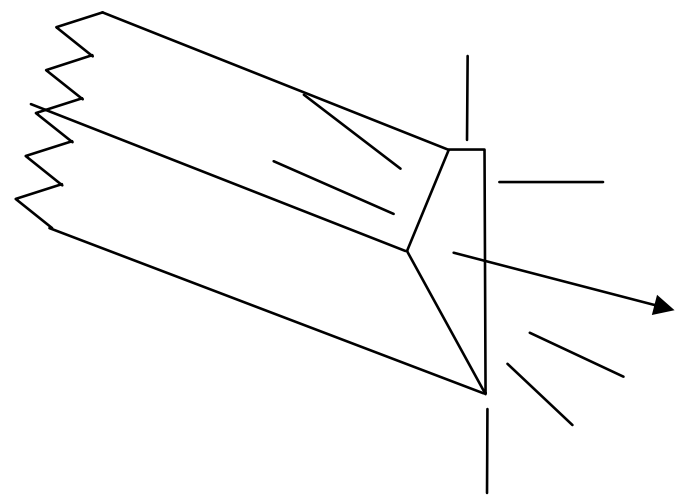

Figure 2. A wave-guide will act as an inefficient radiator

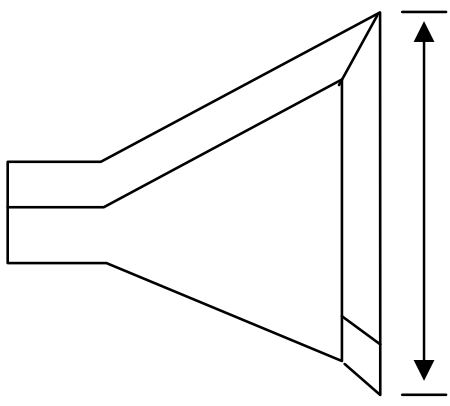

(a) E-plane

Sectoral horn

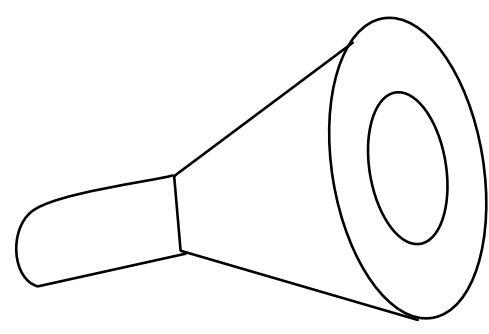

(c) Conical horn

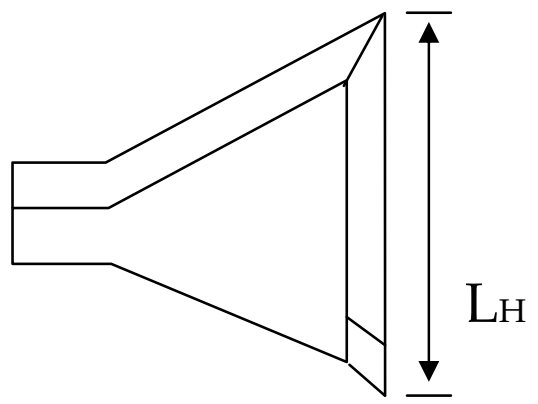

(b) H-plane

Sectoral horn

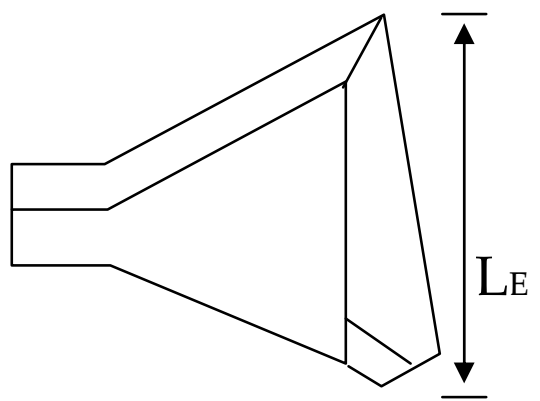

(d) Pyramidal horn

Figure 3. Types of horn Antenna 


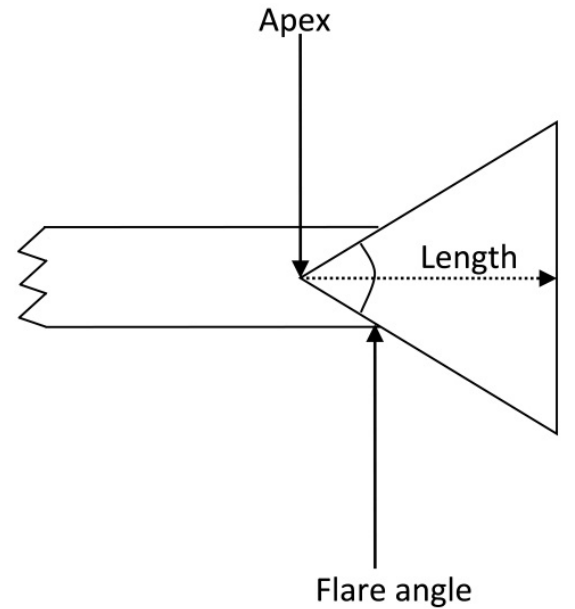

Side view

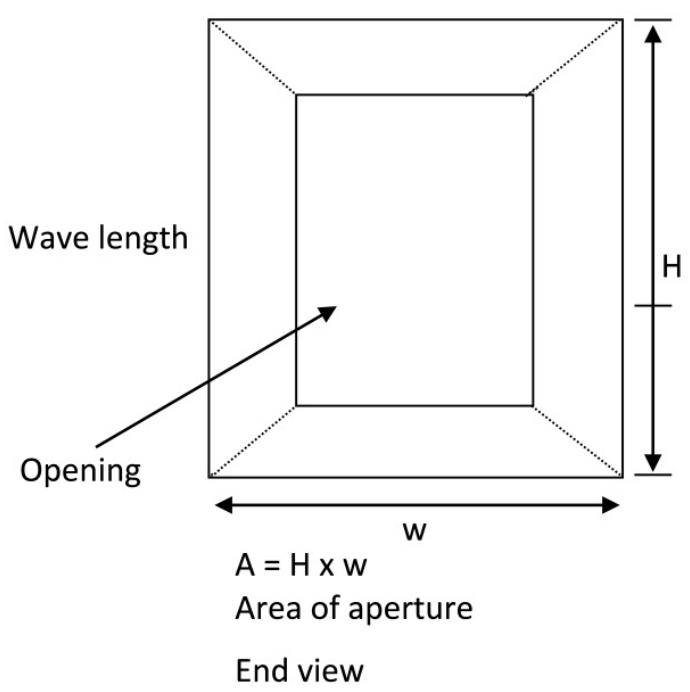

Figure 4. Dimensions of a horn

The important dimensions of the horn antenna are

i. Horn length

ii. Aperture area

iii. Flare angle

The length of a typical horn is usually 2 to 15 wavelengths at the operating frequency. The longer horns though more difficult to mount and work with provide higher gain and better directivity. The aperture area is the area of the rectangle formed by the opening of the horn and is simply the product of the height and width of the horn. The greater this area, the higher the gain and directivity.

The flare angle also affects gain and directivity. Typical flare angles vary from about $20^{\circ}$ to $50^{\circ}$. Increasing the flare angle increases the aperture area. For a given size of aperture area decreasing the length increases the flare angle as shown in Figure 4. Each of these dimensions is adjusted to achieve the desired design objective. An important aspect of a microwave antenna is its bandwidth. Most antennas have a narrow bandwidth because they are resonant at only a single frequency. Their dimensions determine the frequency of operation. Bandwidth is an important consideration at microwave frequencies because the spectrum transmitted on the microwave carrier is usually wide so that a considerable amount of information can be carried. Horn antennas have relatively large bandwidth. Horns are essentially non-resonant or periodic which means they will operate over a wide frequency range.

Horn antennas are used by themselves in many applications but many times higher gain and directivity is desirable. This can easily be obtained by using a horn in conjunction with a parabolic reflector.[1] 


\subsubsection{Parabolic reflector}

The parabolic reflector antenna is one of the most wide spread of all the microwave antennas and is the type that normally comes to mind when thinking of microwaves systems. This type of antenna derives its operation from optics and is possible because microwaves are in transition region between ordinary radio waves and infrared/visible light. The parabolic reflector antenna (often called a dish antenna). The dish is not actually an antenna but serves a reflector. It must be driven by a radiating element at the focal length. This radiating element could be a dipole or a horn radiator. A parabola is a mathematical curve such that its reflection property causes an incoming beam of parallel rays to focus to one point. Conversely radiated waves from a point signal placed at the focal point are reflected by the surface to form parallel rays in the outgoing beam. Thus, a parabolic antenna can be employed as a transmitter and receiving device.

The parabolic reflector may take many forms. The larger the reflector with respect to the wave-length the narrower the beam width.

A typical dish antenna with a paraboloid shape is shown in Figure 5.

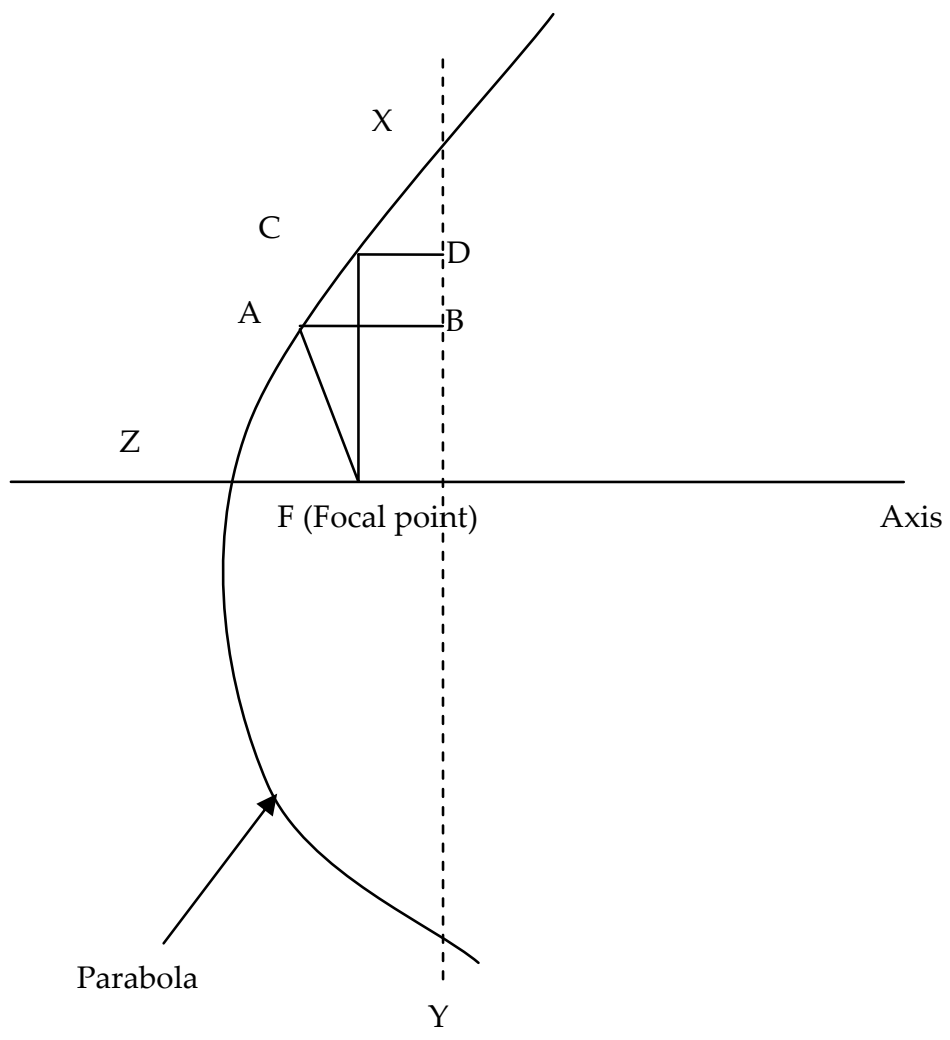

Figure 5. A Parabolic Dish 
A key dimension of the parabola above is a line drawn from its center at point $Z$ to a point on the axis labeled $F$ which is the focal point. The ends of the parabola could extend outwardly for an infinite distance but usually they are limited. The limits are shown by the dashed vertical line with the end points labeled $\mathrm{X}$ and $\mathrm{Y}$. The distance between the focal point and the parabola and the vertical dashed line is a constant value, for example in the Figure 5., the distance represented by the sum of the lines FA to $\mathrm{AB}$ and FC to $\mathrm{CD}$ are equal. This effect causes a parabolic shaped surface to collimate electromagnetic waves into a narrow beam of electrons. Placing an antenna at the focal point $\mathrm{F}$ will cause it to radiate waves from the parabola in parallel lines. If used as a receiver, the parabola will pick up the electromagnetic waves into a narrow beam of electrons. Placing an antenna at the focal point $F$ will cause it to radiate waves from the parabola in parallel lines. If used as a receiver, the parabola will pick up the electromagnetic waves and reflect them to the antenna located at the focal length.

The gain of a parabolic antenna can be determined by:

$$
G=K\left(\frac{\pi D}{\lambda}\right)^{2}
$$

Where $\mathrm{K}$ is the reflection efficiency typically $(0.4-0.7)$

$\mathrm{D}=$ diameter of the dish $(\mathrm{m})$,

$\lambda=$ wave-length $(\mathrm{m})$

The reflection efficiency is 0.6 for most antennas

Thus gain can be approximated as:

The beam width $(-3 \mathrm{~d}$ B) of a uniformity illuminated parabolic reflector antenna is approximated by $B w=\frac{70 \lambda}{D}$.

As stated before horn antenna can be used in conjunction with a parabolic reflector. The Figure 6. ( $a$ and $b$ ) shows how a parabolic reflector is used in conjunction with a horn antenna for both transmission and reception. The horn antenna is placed at the focal point. In transmitting the horn radiates the signal towards the reflector which bounces the waves off and collimates them into a narrow parallel beam, when used for receiving, the reflector picks up the electromagnetic signal and bounces the waves toward the antenna at the focal point. The result is an extremely high gain narrow beam width antenna.

The gain of the horn radiator is proportional to the area (A) of the flared open flange and inversely proportional to the square of the wave-length, $G=\frac{10}{\lambda^{2}}$

Where $\mathrm{A}=$ Flange area $(\mathrm{m})$

$\lambda=$ Wave-length $(\mathrm{m})$

The $-3 \mathrm{~dB}$ beam- width for vertical and horizontal extents can be approximated from the following:

1. Vertical $\varnothing v=\frac{51 \lambda}{b}$

2. Horizontal $\varnothing v=\frac{70 \lambda}{a}$ 


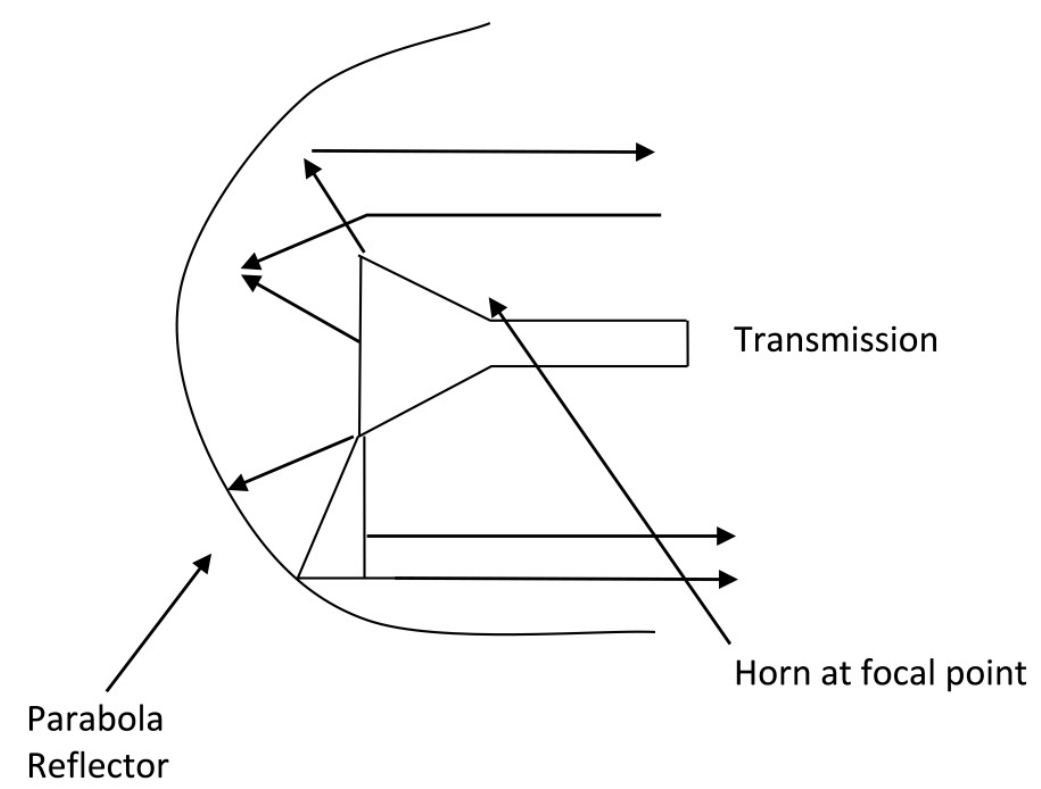

(a) Transmission

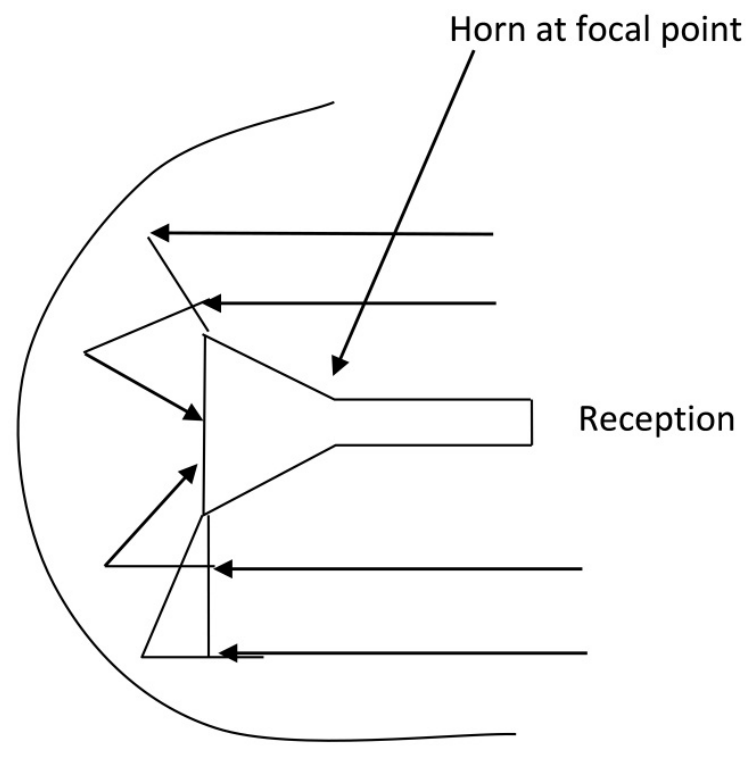

(b) Reception

Figure 6. ( $\mathrm{a}$ and $\mathrm{b})$ Parabolic reflector antenna used for Transmission and Reception 
Where $\varnothing_{\mathrm{v}}=$ Vertical beam width in degrees

$\varnothing \mathrm{h}=$ Horizontal beam width in degrees

$\mathrm{b}=$ Narrow dimension of the flared flange

$\mathrm{a}=$ Wide dimension of the flared flange

$\lambda=$ Wave-length

\subsection{Cassegrain feed system}

A cassegrain reflector system is shown below

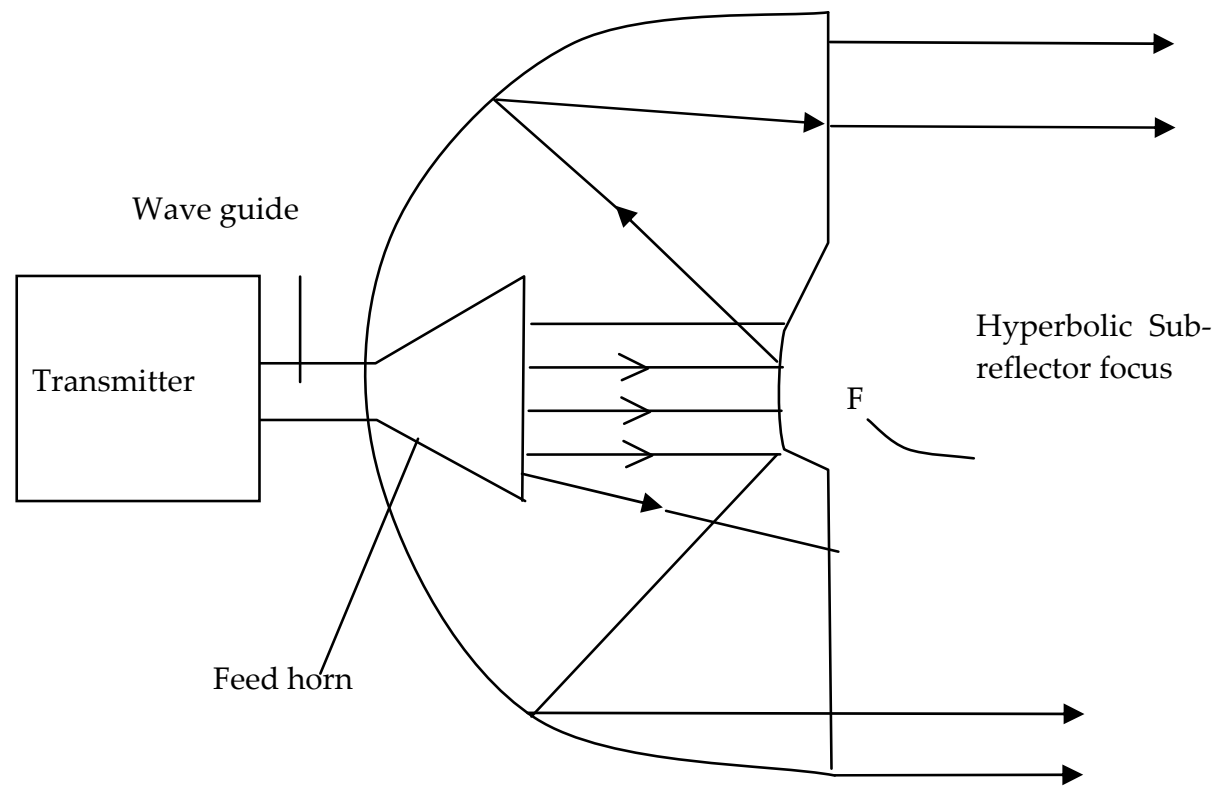

Figure 7. Cassegrain feed parabolic reflector

It consists of a feed horn, a hyperbolic sub reflector and the main parabolic reflector. Radiation from the feed horn illuminates the sub-reflector, which is placed at the focus of the main reflector. Energy scattered by the sub-reflector illuminates the main reflector and produces the secondary radiation pattern at the distant point. This construction leads to a low noise temperature for the antenna.[1]

\subsection{Construction of parabolic reflectors}

Parabolic reflectors are usually constructed of metal panels and the surface finish determines the maximum usable frequency. In many radar systems in order to reduce weight and wind loading and so increase mobility the surface consists of wire mesh. The spacing of the wires must be small compared to the wave-length used so as to reflect most of the incident radiation on the wire mesh. 
Standard parabolic antennas are usually constructed of aluminum. They are manufactured by pressing a sheet of aluminum around a spinning parabola shaped chuck. The reflector themselves are not frequency dependent, but the higher the frequency, the greater the surface perfection required. In practice, the reflectors are therefore specified per frequency band. This antenna has standard parameters of gain, forward/backward ratio (F/B) ratio, beam width, and return loss (RL) if one wants an improvement in these parameters, certain changes to the antenna need to be made.

\section{Antenna properties}

The most important properties possessed by many antennas are polarization, radiation pattern. Directivity and power gain, radiation resistance, band width, effective aperture, power transfer and reciprocity.

1. Polarization: An electromagnetic wave launched from an antenna, may be vertically or horizontally polarized. The direction of the electric field specifies the polarization of the antenna. If the electric field is parallel to the earth electromagnetic wave is said to be horizontally polarized. If the electric field is perpendicular to the earth, the electromagnetic wave is said to be vertically polarized.

Antennas that are horizontal to the earth produce horizontal polarization while antennas that are vertical in the earth produce vertical polarization. For optimum transmission and reception both the transmitting and receiving antennas must be of the same polarization.

Electromagnetic waves are usually vertically polarized though other types of polarization may also be used for specific purposes. Vertical or horizontal polarization is also called linear polarization. Circular polarization refers to a combination of vertical and horizontal polarization.

In a receiving system, the polarization of the antenna and incoming wave need to be matched for maximum response. If this is not the case there will be some signal loss, known as polarization loss. For example, if there is a vertically polarized wave incident on a horizontally polarized antenna, the induced voltage available across its terminal will be zero. In this case, the antenna is cross polarized with an incident wave. The square of the cosine of the angle between wave polarization and antenna polarization is a measure of the polarization loss. It can be determined by squaring the scalar product of unit vectors representing the two polarizations.[2]

2. Radiation Pattern (polar pattern): This is a graphical plot of the field strength radiated by the antenna in different angular directions. The plot may be obtained for the vertical or horizontal polar patterns respectively. A wide variety of polar patterns are possible such as:

a. The omni-directional pattern in which energy is radiated equally in all directions

b. The pencil beam pattern in which energy is concentrated mainly in one direction

c. The multiple beam pattern in which energy is radiated in several adjacent beams. 
The same polar patterns apply whether the antenna is transmitting or receiving radiation because of the principle of reciprocity.

3. Directivity and Gain: The directivity of an antenna is a measure of the ability to direct RF energy in a limited direction rather than in all (spherical) directions equally. The directivity of an antenna refers to the narrowness of the radiated beam. If the beam is narrow in either the horizontal or vertical plane, the antenna has a high degree of directivity in that plane. The power gain of an antenna increases as the degree of directivity increases because the power is concentrated into a narrow beam. The term gain implies that the antenna creates a higher power when it concentrates the power into a single direction. Directivity gain is the gain calculated assuming a loss less antenna in a preferred direction at maximum radiation.[3] Real antennas have losses and power gain is simply the directivity multiplied by the efficiency of the antenna $G=$ D x Eff

Where $G=$ Power gain

$\mathrm{D}=$ Directivity gain

Eff = Antenna efficiency

Power gain is the ratio of the output power of an antenna in a certain direction to that of an isotropic antenna. The gain of an antenna is a power ratio comparison between an isotropic and un-directional radiator. This ratio can be expressed as:

$$
A d B=10 \log _{10} \frac{P_{2}}{P_{1}}
$$

Where $\quad A d B=$ Antenna gain in decibels

$\mathrm{P}_{1}=$ Power of un-directional antenna

$\mathrm{P}_{2}=$ Power reference antenna

4. Radiation Resistance: The radiation resistance is associated with the power radiated by the antenna. It is the portion of an antenna's input impedance that is due to power radiated into space. The power radiated by an antenna is $I^{2} R$ Watts where $I^{2}$ is r.m.s antenna current and $R$, is a fictitious resistance termed the radiation resistance which is a resistance which if it carries the same terminal current as the antenna on transmission will dissipate the same amount of power as the one radiated. The power radiated by an antenna into space have losses in practical antennas, therefore the efficiency is less than $100 \%$. This efficiency can be defined as $E f f=\frac{P_{r}}{P_{T}}$

Where $\operatorname{Pr}_{\mathrm{r}}=$ Radiated power

$\mathrm{P}_{\mathrm{T}}=$ Total power supplied to the antenna.

Recall; that $\mathrm{P}=\mathrm{I}^{2} \mathrm{R}$ we have that $E f f=\frac{I^{2} R_{r}}{I^{2} R_{T}}$

$$
E f f=\frac{R_{r}}{R_{T}}
$$

Where $\mathrm{R}_{\mathrm{r}}=$ Radiation resistance seen at the feed point 
5. Bandwidth: Many antennas which operate at the higher radio frequencies do so over narrow bandwidths of about $10 \%$, because the antennas are resonant at only a single frequency. In recent years attention has been given to designing wide band-width and frequency independent antennas. Wide bandwidths antennas are required to meet the growing demands of telecommunication today.[4,5]

6. Effective Aperture: The power received by an antenna can be associated with a collecting area. Every antenna may be considered to have such a collecting area which is called its effective aperture Ae. If $P_{d}$ is the power density at the antenna and $P_{R}$ is the received power available at the antenna terminals then

$\mathrm{PR}=\mathrm{Pd}$ Ae watts

$$
P_{R}=\frac{P_{R}}{P_{d}} m^{2}
$$

It can also be shown that an antenna with power gain $G$ has an effective aperture. Ae at the operating wave-length which is given by:

$$
A_{e}=\frac{G A^{2}}{4 \pi} m^{2}
$$

7. Power Transfer: For the maximum transfer of power from a receiving antennal to a receiver, the impedance of the antenna should be matched to the input impedance of the receiver in accordance with the maximum power transfer theorem. The maximum power receiver, is given by:

$\operatorname{PR}(\max )=\frac{V^{2}}{4_{m}}$ watts

Where $\mathrm{V}$ is the induced r.m.s

Voltage in an antenna connected to a receiver and $\mathrm{R}_{\mathrm{i}}$ is the input resistance.

8. Reciprocity: The properties of a transmitting antenna are very similar to those of a receiving antenna because of the theorem of reciprocity which states that is an e.m.f is applied to the terminals of an antenna $A$ and it produces a current $i$ at the terminals of antenna B, then the same e.m.f applied to the terminals of antenna B, will produce an equal current $i$ at the terminals

9. side lobes: Microwave antennas are intended to be directional. The maximum radiation is thus in the direction of propagation. In practice, it is impossible to shape all the energy in this direction. Some of it spills out off the side and back of the antenna. Due to the complex phases set up in an antenna pattern, lobes result. The main lobe is around the center of the antenna. Side lobes of lesser amplitude result around the rest of the antenna. The aim of a directional antenna is to maximize the energy in the main lobe by minimizing the energy in the side lobes. It is important to understand the radiation patterns when panning antennas to make sure that one does not pan the signal onto a side lobe.

10. Front-to-back ratio: It is not all energy that is radiated out in the front of the antenna. Some of the energy radiates out of the back lobe. The F/B ratio is defined as the ratio of the gain in 
the desired forward direction to the gain in the opposite direction out of the back of the antenna. It is expressed in decibels. It is very important in microwave radio backbone systems to have antennas with a good $\mathrm{F} / \mathrm{B}$ ratio to enable frequency re-use. Ratios as high as $70 \mathrm{~dB}$ may be required. When specifying the $\mathrm{F} / \mathrm{B}$ ratio of an antenna, a wide angle at the back of the dish should be considered and not just the actual value at 180 degrees.[6]

11. Beam-width: The beam-width is an indication of how narrow the main lobe is. The halfpower beam-width is the width of the main lobe at half power intensity (that is $2 \mathrm{~dB}$ below the bore sight gain): the higher the gain of the antenna, the narrower the beamwidth. The reason has to do with the definition of antenna gain. Recall that as the gain is increased in one direction, the side lobes decrease in another. The beam-width of the antenna is usually decreased by increasing the size of the reflector. High-gain antennas not only improve the fade margin of a radio link but also result in reduced interference from signals off bore sight. One just has to be careful with very high gain antennas that the stability of the towers is sufficient to hold the weight of the large diameter antennas. Towers must also be rigid enough to avoid a power fade from tower twist. It is not uncommon to have microwave antennas with beam-width of less than one degree. With high-gain antennas where the beam-width is very narrow, angle-of-arrival fading can occur. This causes flat fading due to antenna discrimination. In practice, this limits the useful antenna gain especially on very high frequency links.

Beam-width refers to the angle of the radiation pattern over which a transmitter's energy is directed or received. The beam-width is measured on the antenna's radiation pattern. Figure 8. shows the horizontal radiation pattern of a typical directional antenna plotted on a polar coordinate graph.

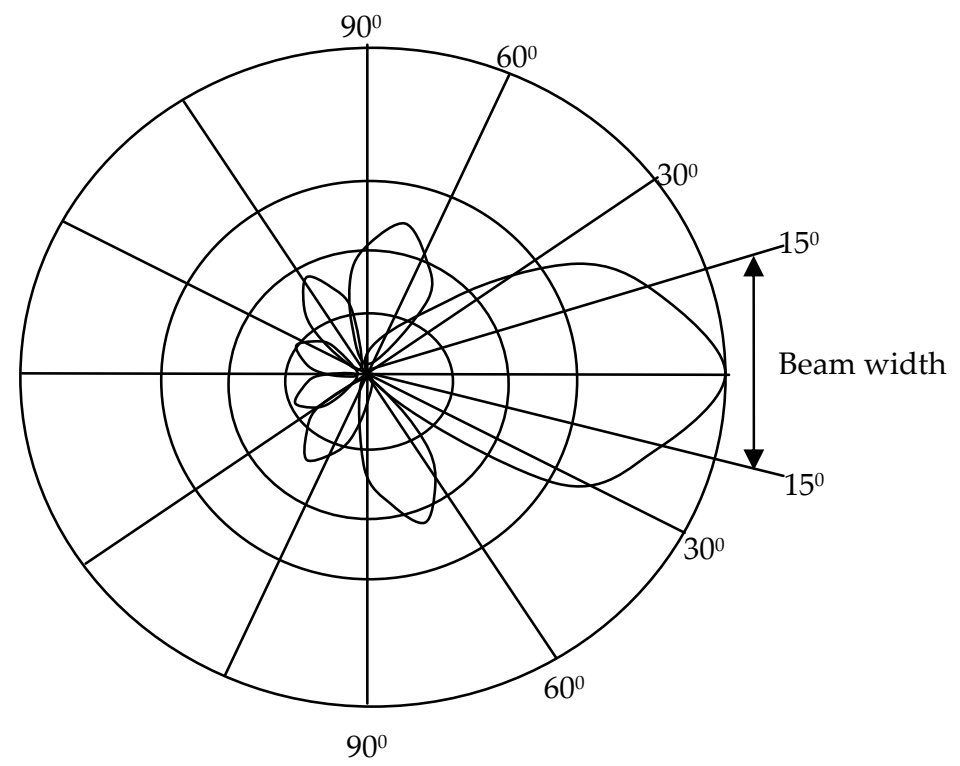

Figure 8. Beam- width 
The antenna is assumed to be at the center of the graph. The concentric circles extending outward from the pattern indicate the relative strength of the single as it moves away from the antenna. The beam- width is measured between the points on the radiation curve that are $-3 \mathrm{~dB}$ from the maximum amplitude of the curve.

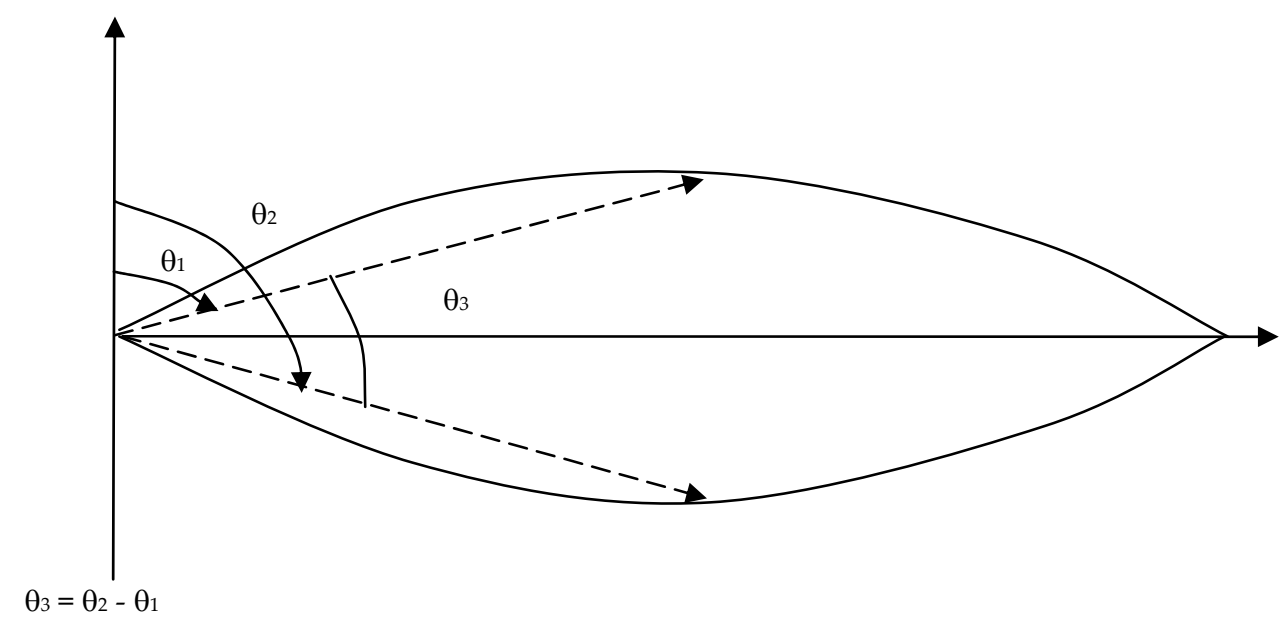

Figure 9. -3dB Beam-width

The beam-width $-3 \mathrm{~dB}$ is the angle subtended at the center of the polar diagram as shown in Figure 9.

\section{Radomes}

Special covers for antennas called radomes are available to protect the horn feed and reduce the wind loading the tower. These radomes vary in their construction depending on the type of antenna. For standard parabolic dishes the radomes are usually a conical shape constructed out of fibre glass. The radome must be constructed such that its insertion loss is minimized.

13. Voltage Standing Wave Ratio: An antenna presents a complex impedance to the feeder system which must be attached to it since the feeder system also represents a fixed impedance, there can be an impedance mismatch at the antenna connection. Not all the power is thus radiated out the antenna. Some power is reflected back down the feeder. This mismatch is quantified in terms of the voltage standing wave ratio (VSWR). In a real system there will always be some mismatch at both ends. A standing wave is therefore set up in the cable from the reflected waves that are reflected up and down the cable. The reflected wave sets up a standing wave with voltage minima and maxima every quarter wave-length. The voltage maxima coincide with points where the incident and reflected waves are in phase and the minima where they cancel in phase. The VSWR can thus be expressed as:[6] 


$$
V S W R=\frac{V_{\max }}{V_{\min }}
$$

The VSWR value will always be greater than unity and the best VSWR is a value that approaches unity. Practically, a good match will result in a value of around 1.2.

A reflection coefficient $(p)$ can be defined that expresses the ratio between the reflected and incident waves.

$$
P=(V S W R-1) /(V S W R+1)
$$

The most convenient way of expressing this mismatch is the return loss (RL), which is the decibel difference between the power incident on the mismatch and the power reflected from the mismatch. The RL in decibels is expressed in terms of reflection coefficient.

$$
R_{L} d B=20 \log \left(\frac{1}{P}\right)
$$

The higher the value of the $\mathrm{R}_{\mathrm{L}}$, the better. Typically, this figure should be better than $20 \mathrm{~dB}$ for microwave radio systems. To achieve this individual components should exceed $25 \mathrm{~dB}$.

\section{Measurement of voltage standing wave ratio}

The VSWR measurements per distance can be determined with the aid of an Anritsu site master instrument. Before the Anritsu site master is used, it has been calibrated as follows:

i. Turn on the site master by pressing the ON button

ii. Press the FREQ soft key from the main menu

iii. Press F1 Soft key from the main menu

iv. Enter the lower frequency limit in $\mathrm{MHz}$ for the antenna system by using the up/down arrow and press ENTER

v. Press the F2 soft key from frequency menu

vi. Enter the higher frequency limit in $\mathrm{MHz}$ or $\mathrm{GHz}$ for the antenna system by using the UP/down arrow and press ENTER

vii. Check that the FREQ $(\mathrm{MHz})$ scale in the display area indicates the new frequency start and stop values.

The next stage in calibration is to perform the following.

i. Press the start key

ii. Choose calibration type and press ENTER

iii. Follow the instruction on the screen.

After the calibration, connect the site master to the transmission line as shown in Figure 10. 
After calibration, the Anritsu site master can now be used for measurement of VSWR. A graph of VSWR versus distance can be plotted using Matlab software program. A VSWR model can be developed using the M-file environment of Matlab Software Program. The data obtained can be curve fitted with polynomial of nth order degree to give a prediction of VSWR per distance on a transmission line. This prediction is necessary for transmission planning purposes.

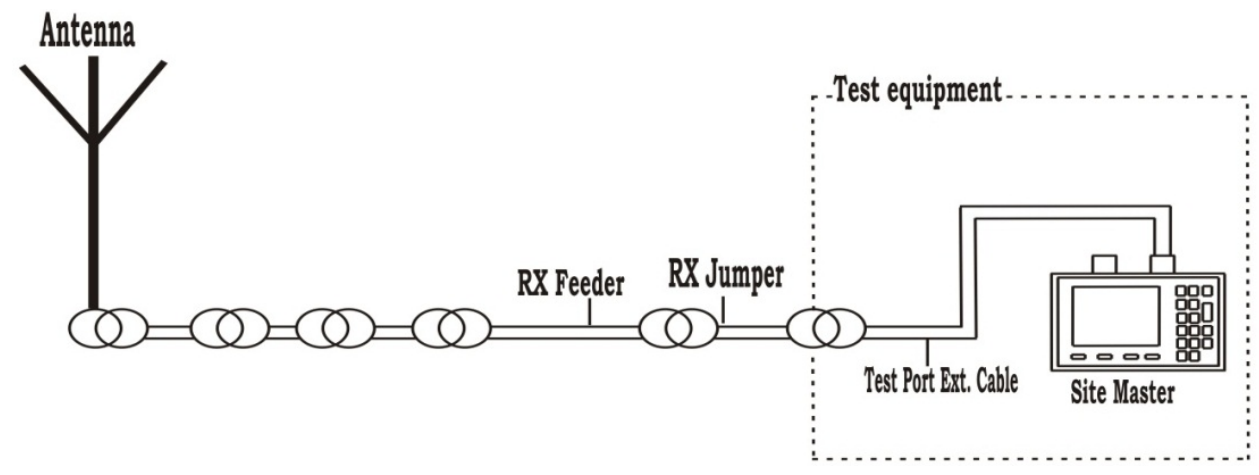

Figure 10. Measurement setup of Anritsu site master instrument

\section{Conclusion}

This chapter is on microwave antenna performance metrics. It describes vividly the types of two most commonly used microwave antennas. It also describes the properties of the antenna. This chapter will be very useful to microwave engineers and planners as a clear understanding of these properties will help in enhancing microwave antenna performance.

\section{Author details}

Paul Osaretin Otasowie

Department of Electrical/Electronic Engineering, University of Benin, Benin City, Nigeria

\section{References}

[1] Frenzel, L.E. (1996). Communication Electronics Macmillan/McGraw-Hill, New York.

[2] Misra, D.K. Radio frequencies and microwave communication circuits and design (2004). John Wiley and Sons Inc. Publication, New Jersey.

[3] Collin, R.E. Foundations of Microwave Engineering (2001) IEEE Press, U.S.A. 
[4] Rappaport, T.S. (2006). Wireless Communication Principles and Practice. Prentice Hall, New Jersey.

[5] Juchen, H.S. (2005). Mobile Communication Addison Wesley Longman Publishing Company Massachusetts, U.S.A.

[6] Manning T.(1999) Microwave Radio Transmission Guide British Library Cataloguing in the Publications data. Uk 